\title{
A Permeameter for Magnetic Testing at Magnetizing Forces up to 300 Oersteds
}

\author{
By Raymond L. Sanford and Philip H. Winter
}

\begin{abstract}
A permeameter is described for magnetic testing at values of magnetizing force up to 300 oersteds. It is designed to test specimens of rectangular cross section up to $3 \mathrm{~cm}$ wide and $1 \mathrm{~cm}$ thick, with a preferred length of $28 \mathrm{~cm}$. The instrument requires only a single specimen and is more simple and rapid to operate than the Burrows permeameter, which for many years has been generally accepted as the standard instrument for magnetic testing in this range. It is an absolute instrument, in that its constants are derived from its own dimensions so that it does not require calibration by any other permeameter. It is estimated that the values of magnetizing force and induction, which are obtained, are accurate within 1 percent, except that for very low values of magnetizing force the precision is the determining factor, and the uncertainty may be of the order of 0.05 oersted.
\end{abstract}

\section{Introduction}

For many years the Burrows Compensated Double-Yoke permeameter, either in its original form, ${ }^{1}$ or as modified by Gokhale, ${ }^{2}$ has been generally accepted as a standard instrument for the magnetic testing of straight bars. As it is an absolute instrument in the sense that its constants are derived from its own dimensions, it does not require calibration by reference to any other permeameter. For this reason, it has usually been employed for the calibration of test bars to be used as reference standards for calibrating and checking the performance of other permeameters.

However, the Burrows permeameter has certain disadvantages. It is somewhat complicated and tedious to operate and requires duplicate specimens, one of which is used as an auxiliary. Furthermore, it is extremely sensitive to the effect of magnetic nonuniformity along the length of the specimen which leads to errors which are indeterminate and may be large. Consequently, its use in recent years has been limited in general to the testing of magnetically uniform bars to be used as standards for calibration purposes.

For some time there has been a need for an

${ }^{1} \mathrm{C} . \mathrm{W}$. Burrows, The determination of the magnetic induction in straight bars, Bul. BS 6, 31 (1909) S117.

${ }^{2}$ U. S. Patent $1,559,085$ (1925)。 absolute permeameter that would have an accuracy comparable with that of the Burrows instrument but which would be less complicated to operate. For this reason the development of such an instrument was undertaken. The result is the $\mathrm{MH}$ permeameter, ${ }^{3}$ designed to operate at magnetizing forces up to 300 oersteds. It is capable of testing specimens of rectangular cross section up to $3 \mathrm{~cm}$ wide and $1 \mathrm{~cm}$ thick, with a preferred length of $28 \mathrm{~cm}$, although specimens $24 \mathrm{~cm}$ in length can be tested satisfactorily. It is absolute in that its constants can be derived from its own dimensions, so that it does not require calibration by any other permeameter. It is estimated that values of either magnetizing force or induction can be determined to within 1 percent, except that for very low values of magnetizing force, the uncertainty due to lack of precision may be of the order of 0.05 oersted. It is simple and rapid to operate and requires only a single specimen.

\section{Description of the Permeameter}

The permeameter is shown in figure 1. The specimen, of rectangular cross section, is surrounded by the main magnetizing coil. The magnetic circuit is completed by symmetrical

\footnotetext{
3 The designation $\mathrm{M} H$ stands for medium $H$ (magnetizing force).
} 
$U$-shaped yokes that are supported by end pieces, which in turn are supported by four Bakelite blocks. These end pieces also support the specimen and provide for close contact between the yoke and the specimen. The symmetrical yoke structure is of advantage because it promotes a uniform cross-sectional distribution of magnetic flux in the specimen. This is especially important in determining points on the hysteresis loop where the magnetizing force and the induction are in opposite directions. Auxiliary magnetizing coils are located around the ends of the yokes and supporting end pieces that make contact with the specimen. These auxiliary coils are of advantage because they promote a uniform longitudinal distribution of flux in the specimen by overcoming the contact reluctance between the specimen and the yoke. The flux distribution in the region adjacent to the center section of the bar is thereby improved, and since the value of the magnetizing force is obtained by measurements in this region, the accuracy of the measurements depends upon the degree of uniformity that can be obtained.

Values of flux-density, $B$, are obtained by means of a test coil surrounding the bar at the middle. An auxiliary test coil whose area-turn value is adjusted to be equal to that of the $B$-coil is located adjacent to the $B$-coil, but not surrounding the specimen, and connected in series opposing the $B$-coil. Thus the galvanometer deflections observed are a measure of the intrinsic flux density, $B_{i}$, rather than the total flux density. This obviates the necessity of calculating the air-flux correction for bars of different cross-sectional area.

Values of magnetizing force, $H$, are obtained by means of $H$-coils so mounted that they can be made to rotate $180^{\circ}$ in the region adjacent to the

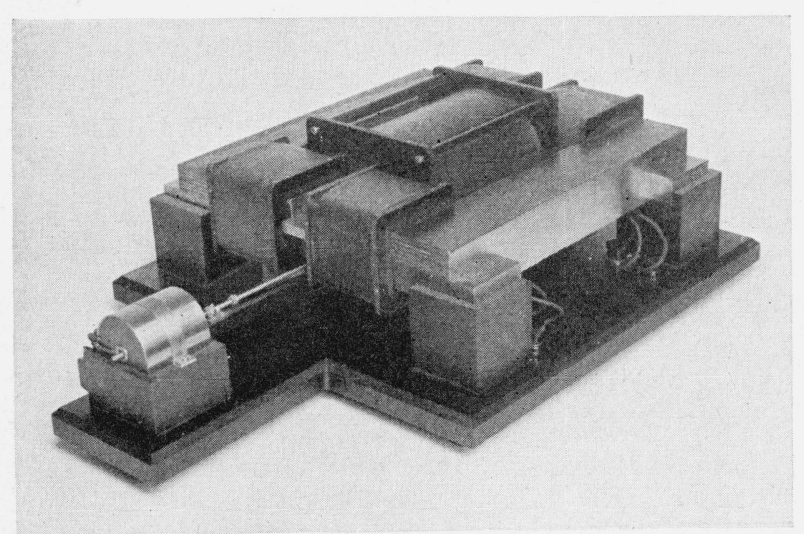

Figure 1. The MH permeameter. center section of the bar. There are two $H$-coils mounted one above the other on a rotating brass table and adjusted to equal area-turn values. When these coils are connected in series, opposing, with their axes parallel to the specimen, and rotated through $180^{\circ}$, the galvanometer deflection observed is proportional to the gradient of the field with respect to the distance from the surface of the specimen, which will be referred to as the radial gradient. The value of $H$ as measured by the deflection when only the upper coil is connected to the galvanometer is extrapolated to the surface of the specimen by using the observed value of the radial gradient.

The use of rotatable $H$-coils, or "flip-coils," has been found to have certain definite advantages over the use of stationary $H$-coils. Identity in the observed values of magnetizing force for both directions of the magnetizing current is a good criterion of a satisfactorily demagnetized condition. Also, values of magnetizing force for points on the hysteresis loop can be obtained by direct observation instead of by calculation in terms of the difference between two large quantities. This improves both the precision and the accuracy of the measurements.

The magnetic circuit is shown in detail in figure 2. The laminated yokes are of No. 16 gage silicon sheet of armature grade and are 4 by $5 \mathrm{~cm}$ in cross section. The specimen rests on end pieces that extend between the supporting Bakelite blocks. This provides a good magnetic contact between the yokes and the flat surface of the specimen at the ends. The ends of the U-shaped sections are recessed to fit over and slide on the end pieces so that contact is made at the edges of the specimen. This construction provides good contact between the yokes and the specimen without the necessity of clamping. This is an important feature, especially when strain-sensitive materials are to be tested. A supporting platform associated with the test-coil assembly prevents strains that might result from sagging of the specimen. The over-all length of the yokes is $28 \mathrm{~cm}$, and the specimen is preferably of this length. However, bars as short as $24 \mathrm{~cm}$ can be tested if necessary.

The main magnetizing coil is $13.8 \mathrm{~cm}$ long, wound on a Bakelite form whose inside diameter is $7 \mathrm{~cm}$. The winding is of No. $18 \mathrm{AWG}$ Formexinsulated magnet wire and has 1,540 turns uniformly wound in 12 layers. The four compensat- 


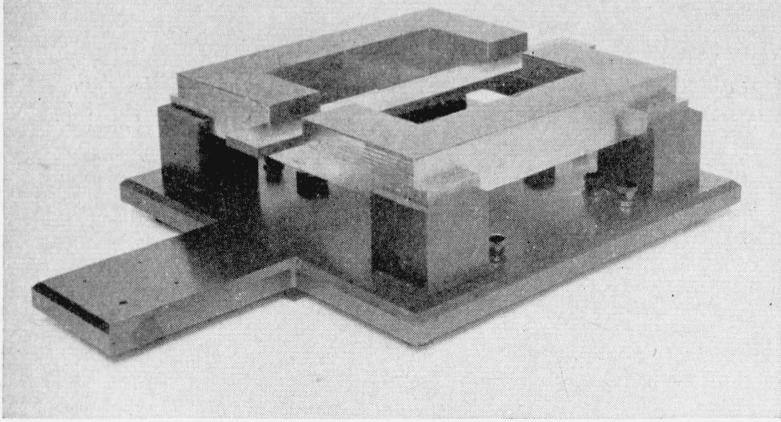

Figure 2. Magnetic circuit of the $M H$ permeameter.

ing coils on the yokes are $5.8 \mathrm{~cm}$ long, and each has 430 turns of No. 18 Formex-insulated wire wound in eight layers. As it was desired to avoid the complication of having to adjust the compensating current for each point to be determined, the effect of the compensating coils on the degree of uniformity of the field adjacent to the specimen was determined for various ratios of the number of turns in the main and compensating coils. Although perfect compensation could not be obtained over the whole range for a single ratio of turns, that ratio which appeared to give the best results on the average was adopted and the coils were connected in series with the main magnetizing coil. Observations of the radial gradient were then made for different types of specimen as related to the value of magnetizing force. It was found that, within the experimental error, the percentage correction to the magnetizing force varies linearly with the permeability of the specimen, as shown in figure 3 . It was also found that, although under some conditions the gradient was not quite linear, the variation from

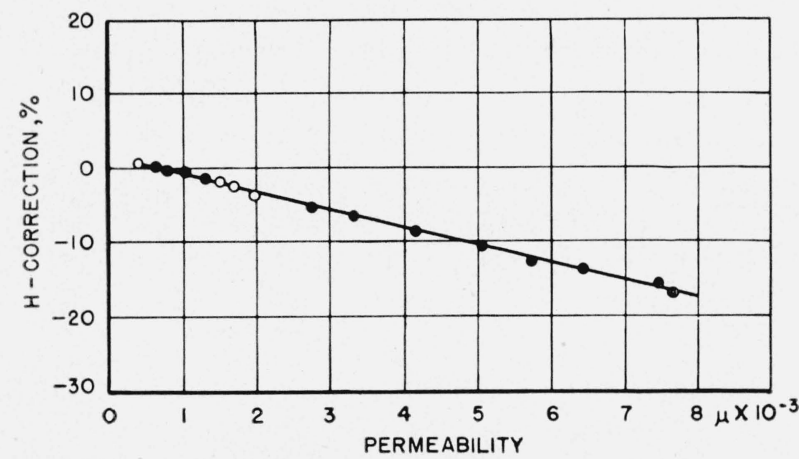

FIGURE 3. Relation of H-correction to permeability.

Open circles relate to cold-rolled carbon steel. Closed circles relate to silicon steel. linearity was so small that no significant error is introduced from this cause.

The test-coil assembly is shown in figure 4 . It is held in position within the main magnetizing coil form by Bakelite rings. The platform extending between these rings supports the specimen and is recessed at the middle so that the upper $H$ coil can be located close to the surface of the specimen. The two $H$ coils are mounted below the specimen on a small table that is rotated by a horizontal rod through a rack and pinion gear by an iron-clad solenoid and plunger located far enough away from the magnetic circuit to avoid interference. Adjustable stops limit the rotation of these "flipcoils" to $180^{\circ}$. The upper coil has an area-turn value of approximately $8,250 \mathrm{~cm}^{2}$-turns. The lower coil has a slightly greater area-turn value and is shunted with a resistance so as to make its effective value equal to that of the upper coil. Each coil is approximately $2.9 \mathrm{~cm}$ long, $4.44 \mathrm{~cm}$ wide, and $1.11 \mathrm{~cm}$ thick. The axes of the coils are $1.11 \mathrm{~cm}$ apart, and the axis of the upper coil is $0.87 \mathrm{~cm}$ from the surface of the specimen. The windings are of No. 34 Formex-insulated magnet wire wound on Micarta forms.

The $B$-coil, of the same length as the $H$-coil, is wound on a Micarta form and surrounds the specimen. It has 100 turns of No. 31 single silk and enamel-insulated wire. The inside dimensions are such as to accommodate a specimen $3 \mathrm{~cm}$ wide and $1 \mathrm{~cm}$ thick. The compensating coil of the same area-turn value as the $B$-coil is mounted above it and is connected in series, opposing.

The usual switches and rheostats are employed to control the magnetizing current supplied by a storage battery. The measurements are made with a heavily overdamped ballistic galvanometer calibrated by means of a standard mutual inductor in the regular way. ${ }^{4}$

\section{Performance}

The use of a symmetrical yoke structure promotes uniformity of flux distribution, particularly across the section of the specimen. However, magnetic leakage due to the presence of joints in the magnetic circuit causes a certain lack of uniformity of the field near the surface of the specimen in the region occupied by the $H$-coils. Many tests were made to determine whether or

${ }^{4}$ NBS Circular C456, p. 23 (1946). 


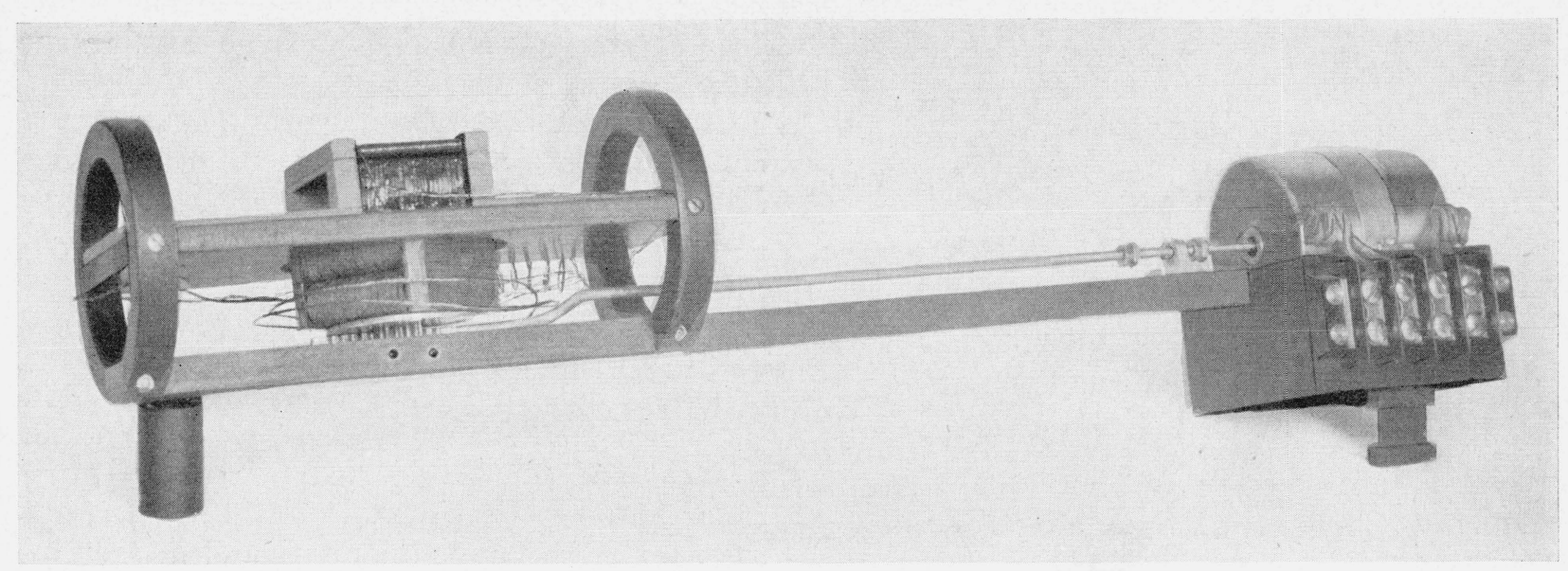

Figure 4. Test-coil assembly.

not properly located auxiliary magnetizing coils would be effective in improving the distribution. From a survey of the leakage flux around the magnetic circuit it was determined that auxiliary magnetizing coils placed on the end sections of the yokes would greatly improve the flux distribution in the region where the measurements were to be made. Distribution tests were then made with various specimens with and without the auxiliary coils. It was found that with the auxiliary coils the longitudinal distribution of the flux in the region adjacent to the center section of the specimen was uniform and, with the use of a double $H$-coil to determine the radial gradient, the value of magnetizing force at the surface of the specimen could be determined with satisfactory accuracy.

It is generally recognized that the degree of magnetic uniformity of the specimen affects the results of a magnetic test. The nature and magnitude of the effect depends not only upon the nature and magnitude of the nonuniformity but also upon the characteristics of the testing apparatus. In the $\mathrm{MH}$ permeameter, the test is limited to the middle $3 \mathrm{~cm}$ of the bar. It is conceivable of course that extreme nonuniformity in the part of the bar near the middle might affect the observed value of magnetizing force unduly, but such a condition is relatively rare. However, valid comparisons between permeameters that include different lengths of the specimen in the test cannot be made unless the test specimens are uniform.

A source of uncertainty often ignored, but which should not be disregarded, is the effect of mechani- cal strain. It is well known that the high-permeability alloys, especially in sheet form, are likely to be exceedingly sensitive to strain. However, the effect of strain in solid materials of other types is often overlooked. For instance, in a recent test of a solid bar that was not quite straight, the induction corresponding to a given value of magnetizing force could be changed by as much as 2 kilogausses by varying the clamping pressure. To avoid this uncertainty, the $\mathrm{MH}$ permeameter was so designed that good contact between the yokes and the specimen could be obtained without the necessity of clamping.

In estimating the accuracy of any permeameter, it is necessary to take into account not only the sources of error peculiar to the individual apparatus, but also those inherent in ballistic methods in general. The factors inherent in ballistic methods include the value of the mutual inductor, the area-turns of the test coils, the adjustment of the galvanometer sensitivity, ability of the galvanometer to integrate the value of a delayed impulse correctly, temperature effects, degree of uniformity of the specimen, and observation errors. The principal points peculiar to the $\mathrm{MH}$ permeameter are the distribution of the magnetic field in the space adjacent to the specimen occupied by the $H$-coils and the longitudinal and transverse distribution of the magnetic flux within the specimen.

The value of the standard mutual inductor has been determined to within \pm 0.05 percent by comparison with an absolute standard of high accuracy.

The area-turns of the $H$-coil have been deter- 
mined by a method previously described ${ }^{5}$ with an estimated accuracy of \pm 0.2 percent.

The current in the mutual inductor for calibrating the galvanometer is easily adjusted to within \pm 0.05 percent by using a potentiometer and a standard shunt.

Recent theoretical and experimental studies of the behavior of the ballistic galvanometer in use with this permeameter have shown that under the most severe conditions likely to be encountered, the galvanometer integrates correctly to within 0.1 percent.

In view of the fact that the specimen is not closely surrounded by the magnetizing coil and that the currents are relatively low, the heating is negligible under normal conditions.

With the understanding that the test results relate only to the middle $3 \mathrm{~cm}$ of the specimen, errors due to nonuniformity along the length of the specimen are usually of negligible magnitude.

The observational error of a single reading may be as much as $0.2 \mathrm{~mm}$. However, by taking a sufficient number of readings, the probable error can be reduced to about $0.1 \mathrm{~mm}$. With suitable adjustment of the galvanometer sensitivity, this will correspond to not over 0.2 percent for values of magnetizing force of 5 oersteds or over, but for values of less than 5 oersteds the precision is the determining factor and the uncertainty is of the order of 0.05 oersted. The observational error in the measurement of the induction can easily be kept within 0.2 percent by suitable adjustment of the galvanometer sensitivity.

The accuracy of the observed values of magnetizing force is limited mainly by the degree of uniformity of the field in the space occupied by the $H$-coils and adjacent to the specimen. In order to obtain a reasonable degree of precision, it was necessary to choose dimensions for the test coils that represent the best compromise between the conditions for sensitivity and for accuracy. From the results of the experimental exploration of the field, it is estimated that errors due to nonuniform distribution do not exceed 0.3 percent.

Experimental evidence indicates that the longitudinal and transverse distributions of flux within the part of the specimen covered by the $B$-coil are sufficiently uniform to make errors from this source negligible.

For purposes of comparison, normal induction

${ }^{5}$ NBS Circular C456, p. 24 (1946).

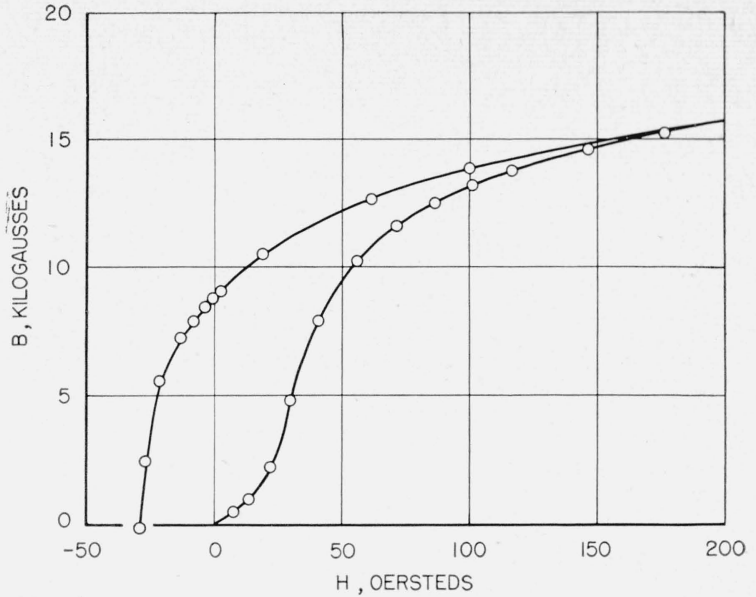

Figure 5. Normal induction and hysteresis curves for tungsten-steel bar.

Curves represent data obtained with solenoid. Check points represent data obtained with $\mathrm{MH}$ permeameter.

and hysteresis tests were made on a tungsten steel bar about $94 \mathrm{~cm}$ long with a rectangular crosssectional area of about $1.8 \mathrm{~cm}^{2}$. After confirming the magnetic uniformity of the bar, it was first tested in a long solenoid and then in the $\mathrm{MH}$ permeameter. Before testing the bar in the solenoid, the distribution of flux density in the center region of the solenoid was checked, and it was found that the radial gradient of $H$ was very small, so that values of $H$ could be obtained with a single flat $H$-coil with an error due to this gradient of less than 0.2 percent.

The curves in figure 5 are from values obtained with the solenoid, and the check points are plotted from values determined with the $\mathrm{MH}$ permeameter. A comparison of the curves and check points shows that the discrepancy between the values determined with the solenoid and $\mathrm{MH}$ permeameter is less than the observational error and well within the normal tolerance.

Considering all the probable sources of error, it is estimated that by the exercise of care in taking the readings and averaging several readings for each point, values of induction can be obtained that will be accurate to within about 0.5 percent, and values of magnetizing force can be obtained that will be accurate to within 0.5 percent, or 0.05 oersted, whichever is larger. For routine tests, values of either induction or magnetizing force accurate to within 1 percent should be obtained without difficulty.

Washington, March 9, 1950. 